${ }^{1} \mathrm{E}$ Çakır, ${ }^{2} \mathrm{E}$ Torun, ${ }^{3} \mathrm{~N}$ Büyükpınar, ${ }^{2} \mathrm{AH}$ Gedik, ${ }^{4} \mathrm{~S}$ Ziyade, ${ }^{5} \mathrm{HNS}$ Duru, ${ }^{6} \mathrm{M}$ Bilgin, $U$ Topuz. 'Department of Pediatric Pulmonology; ${ }^{2}$ Department of Pediatrics, ${ }^{3}$ Department of Pathology; ${ }^{4}$ Department of Chest Surgery, Bezmialem Vakif University; ${ }^{5}$ Department of Pediatrics, Haseki Educational and Research Hospital; ${ }^{6}$ Department of Radiology; 'Department of Anesthesiology, Bezmialem Vakif University, Istanbul, Turkey

Pulmonary nocardiosis is a rare respiratory infection which commonly affects immunocompromised patients but also immunocompetent hosts. The clinical manifestation is variable and endobronchial nocardiosis is indeed a very rare condition. We report a case of endobronchial nocardiosis presenting as nonresolving pneumonia and lung abscess in an immunocompetent child. No predisposing factor could be shown for Nocardia infection. To the our knowledge, this is the first case of endobronchial nocardiosis in the childhood period reported in the literature.

\section{PREDICTION OF MORTALITY CIRCUMSTANCES IN THE PEDIATRIC INTENSIVE CARE UNIT}

doi:10.1136/archdischild-2012-302724.1719

${ }^{1} \mathrm{~N}$ Hazhir, ${ }^{2} \mathrm{R}$ Ghergherehchi. 'Medical Faculty; ${ }^{2}$ Tabriz University of Medical Sciences, Tabriz, Iran

In pediatric ICU(PICU),retrospective studies done during the past decade, indicate that $40-60 \%$ of all deaths follow limitation or withdrawal of life sustaining treatment. We aimed to describe mode of death and the circumstances surrounding dying a pediatric intensive care unit. A retrospective descriptive study all patients $(<15$ years) dying in the PICU of tertiary care hospital $(n=74)$. Information regarding sex, age, Length of Stay (LOS), primary and admission diagnosis and the way of death was determined. Deaths were classified in 5 groups: Do not resuscitate (DNR), Withdrawal or Limitation of Therapy (W/LT), failed cardiopulmonary resuscitation (Failed CPR), brain death (BD) and terminal organ failure (TOF). Among 1075 admission, 6.8\% patients died. Afton admitted during evening (43\%). $40.8 \%$ died in the first two days. Failed CPR was the most common mode of death (66.2\%), BD was found in $14.9 \%$, TOF in $12.2 \%$, W/LT in $2.7 \%$ and DNR in $4.1 \%$. We observed that failed CPR is the most common mod of death and active withdrawal is still not widely practiced in our PICU because pediatricians in developing countries have to consider socio cultural and religious factors when making such decisions.

\section{0 "SNIPPING OF A TONGUE TIE" IN NEONATES WITH ANKYLOGLOSSIA AND BREASTFEEDING PROBLEMS: OUTCOMES AND COMPLICATIONS}

doi:10.1136/archdischild-2012-302724.1720

'E Post, ${ }^{2} \mathrm{~J}$ Daamen, 'W Balemans. 'Pediatrics, Sint Antonius Ziekenhuis, Nieuwegein; ${ }^{2}$ Medicine, University Medical Center Groningen, Groningen, The Netherlands

Background and Aims Breastfeeding is considered the golden standard in neonatal nutrition. One of the complications encountered in breastfeeding is tongue-tie (ankyloglossia), which may eventually lead to aborting breastfeeding. Frenotomy is considered a harmless and effective procedure for ankyloglossia. However, different researchers question the positive effects and emphasize the possible complications, especially when the procedure is performed without general anaesthesia. Aim of our study is to describe the outcomes of frenotomy in neonates.

Methods The data of a series of 166 breastfed neonates (110 male, 56 female) with ankyloglossia and breastfeeding problems under 3 months of age were collected. In these neonates frenotomy was performed between January 2008 en 2012 by an experienced paediatrician in the outpatient clinic without general anaesthesia. Parent(s) attended the procedure and (breast)feeding was given within minutes after frenotomy. One week after frenotomy, data on complications and effects on breastfeeding were collected by a telephone interview with one of the parents.

Results 34 Neonates (20\%) were lost to follow-up after frenotomy. Of the remaining 132 neonates, 117 (89\%) reported improvement in breastfeeding (better latch, less nipple pain or fully breastfed) 12 (9\%) Reported no improvement. Improvement was controversial in 3 neonates (2\%) because of additional problems affecting breastfeeding. Minor complications were reported in 5 patients $(4 \%)$. These consisted of need for a mild analgesic or minimal bleeding up to 1-2 minutes. No major side effects were reported.

Conclusions Frenotomy without general anaesthesia is a safe and very effective procedure in neonates with tongue-tie experiencing breastfeeding problems.

\section{ANALGESIC EFFECT OF A VIBRATION DEVICE ON VENIPUNCTURE IN CHILDREN}

doi:10.1136/archdischild-2012-302724.172

'S Aydinoz, 'FA Genc, ' $G$ Aydemir, 'F Celikel, ${ }^{2} \mathrm{RG}$ Sezer, 'S Suleymanoglu. 'Pediatrics, GATA Haydarpasa Teaching Hospital; '2Pediatrics, Zeynep Kamil Maternity and Childrens' Diseases Training and Research State Hospital, Istanbul, Turkey

Background and Aims Venipuncture is a frequent source of painful procedures for children and it has been well documented that children react to pain with a combination of physiologic and behavioral responses. It has been known that children are unable to describe pain and at particularly high risk for inadequate pain management. FLACC scale is a widely used pain assessment tool for measurement of pain infants and young children. FLACC assess 5 behavioral parameters including facial expression, leg position, activity, crying and consolability. Vibration Anesthesia Device ${ }^{\mathrm{TM}}$ (VAD) is a device specifically designed for management of pain. The objective of this study was to investigate the efficacy of VAD on pain scores assessed with FLACC during and after venipuncture procedure.

Methods Study participants were 60 healthy children undergoing venipuncture procedure for routine laboratory tests. Children were divided into two groups as follows: Group $1(\mathrm{n}=30)$ were placed vibration anesthesia device 5 to $10 \mathrm{~cm}$ proximally through the site of venipuncture and group $2(n=30)$ underwent venipuncture only. A single observer rated pain responses using FLACC before, during and after the procedure.

Results Groups did not differ by age and sex. There were no differences between pain scores of groups assessed by FLACC scale before, during and after venipuncture procedure.

Conclusions We assessed the efficacy of a vibration anesthesia device and our results suggested that this device did not reduced pain scores in children during and after venipuncture procedure.

\section{2 METABOLIC SYNDROM: BIRTH WEIGHT AND CHILDHOOD OBESITY}

doi:10.1136/archdischild-2012-302724.1722

Z Yazdanpanahi, M Hajifoghaha, A Nematollahi. Nursing, Shiraz University of Medical Sciences, Shiraz, Iran

Background Obesity is a worldwide health problem at all ages of life span. A large number of research have shown a positive association between Birth Weight(BW), child hood obesity and Metabolic Syndrome in later life, and thus the prevention is critical.

Material and Methods This article presents the results of a systematic review of the association between Birth weight, childhood obesity and Metabolic syndrom.

Result Several studies pointed out that BW and child hood obesity have contributed to an increase in the prevalence of MS in obese pediatric populations born large gestational age (LGA) and macrosomia. Persistent fetal obesity during infancy and child hood rise the 
risk of obesity and serious adult health problem such as cardio vascular disease, diabetes cancer, hypertension, psychological problem, arthritis, arthrosclerosis and diminished physical abilities.

Conclusion Due to dramatic increase in epidemiology of obesity and related health problem we have to prevention of it from fetal period and so we should start it before pregnancy with controlling intervention causes of obesity in mother and provide appropriate educating and counseling. Furthermore follow up care during pregnancy infancy and child hood is needed to reduce risk of diseases that has relation to obesity.

\section{NEWBORN AUTOPSIES: EXPERIENCE OF A REFERRAL LEVEL III NEONATAL INTENSIVE CARE UNIT IN TURKEY}

doi:10.1136/archdischild-2012-302724.1723

${ }^{1} \mathrm{H}$ Tatar Aksoy, ${ }^{1} S S$ Oguz, ${ }^{1} \mathrm{~N}$ Uraş, 10 Ö Erdeve, ${ }^{2} \mathrm{H}$ Bayramoğlu, ${ }^{2} \mathrm{~S}$ Zergeroğlu, ${ }^{3} \mathrm{U}$ Dilmen. 'NICU, Zekai Tahir Burak Maternity and Teaching Hospital, Department of Neonatology; ${ }^{2}$ Pathology, Zekai Tahir Burak Maternity and Teaching Hospital; ${ }^{3}$ Zekai Tahir Burak Maternity and Teaching Hospital/YIldırım Beyazıt University Department of Pediatrics, Ankara, Turkey

Aim Neonatal autopsies is a guide to explore the causes of the perinatal mortalities which is important marker for evaluation of the health policies. Multidisciplinary approach which includes obstetrician, pediatrician, pathologist and geneticist is required for the neonatal autopsies. We aimed to evaluate the neonatal autopsies in our center.

Methods Thirty-eight neonatal autopsies performed between January 2009 and December 2010 were evaluated in respect to demographic characteristics, clinical and pathological diagnosis retrospectively.

Results 7055 neonates were administered to our NICU between January 2009 and December 2010. 404 of the neonates passed away $(5.7 \%)$. Only the $38(9.4 \%)$ of the neonates' parents give permission for autopsy. Of these neonates were 15 female (39\%) and 23 male (61\%). Sixty percent of these neonates were premature. Prematurity was higher in male neonates ( $\mathrm{p}=0.001)$. Median gestational age was 32 (22-41) weeks. Median overall survival of the neonates were 4 (0-80) days. Eighty-three percent of the clinical diagnoses were correlated with the pathological diagnosis. Sixty percent of the clinical and pathological diagnoses were cardiovascular anomalies, diaphragmatic hernia, perinatal asphyxia and prematurity. Two neonates had pneumonia diagnosis by the autopsy. Only one of the neonate's placenta had chorioamnionitis.

Conclusion Neonatal autopsy rates should be increased to decrease the neonatal mortality rate in our country. Neonatal autopsies should be done with multidisciplinary approach and become prevalent and get more progress in our country.

\section{INEXPENSIVE HOMEMADE AGAR-BASED MODELS FOR ULTRASOUND-GUIDED CENTRAL VENOUS CATHETERIZATION TRAINING PROGRAMS}

doi:10.1136/archdischild-2012-302724.1724
N Pozzi, A Scoppa, L Orfeo. Neonatal and Pediatric Intensive Care Unit, 'G. Rummo' Hospital, Benevento, Italy

Background and Aims Central venous catheterization (CVC) plays a central role in patient management in intensive care settings. Compared with the use of traditional anatomical landmarks, ultrasound (US)guidance is associated with higher success rate and fewer mechanical complications. In order to implement the use of US-guided CVC in our Neonatal and Paediatric Intensive Care Unit, we organized a hands-on training program based on the use of agar-handmade models.

Methods Two different models were constructed to simulate vessels, as described by S. Di Domenico et al in Journal of Ultrasound (2008). In model A the vessels were visualized under a flat surface in both transverse and longitudinal scans, whereas in model B the vessels were punctured under a curved surface and the Doppler function was demonstrated. The training session began with a 40-minute lecture followed by the hands on session. We trained 10 paediatric intensivists. Each test was considered completed when participants were able to position the needle correctly on the "first attempt".

Results $60 \%$ of trainees correctly positioned the needle at the first test on model A, whereas only $20 \%$ on model B because of the more complicated technique. The percentage of participants who achieved correct needle position increased steadily with repeated punctures showing a quick acquisition of the basic skills of US-guided puncture.

Conclusions Agar-based models are useful tools for teaching the basic hand-eye coordination skills of ultrasound-guided CVC thus reducing hazardous attempts on real patients and facilitating the introduction of this technique in clinical practice.

\section{SUCCESSFUL MEASURES TO DECREASE HOSPITAL LENGTH OF STAY IN GENERAL PAEDIATRC WARD}

doi:10.1136/archdischild-2012-302724.1725

MM Abou Al-Seoud, IS Ahmed, SS Adham. Paediatric Department, King Fahad Armed Forces Hospital, Jeddah, Saudi Arabia

\section{ELOS $=$ Expected LOS ALOS $=$ Average LOS}

Background and Aims With the increasing demands on health care services a reduction in hospital length of stay (LOS) became a necessity. The number of patients admitted to the 40 beds paediatric ward at KFAFH has increased by $40 \%$ in 2011 . The department has taken measures to optimize beds utilization.

Methods ALOS for the commonest diseases requiring admissions was calculated retrospectively for the year 2007 and was found to be higher than the reported values.

The following steps were implemented:

- Increase awareness and feedback in January 2008

- Update Practice Guide lines in April 2008.

- Multidisciplinary Discharge Planning team in March 2009.

- Consultant profiling in October 2010.

- Forms for prolonged LOS justification in November 2010

- ELOS included in history and physical forms in October 2011

- Cards for ELOS in November 2011.

Abstract 1725 Table 1 Average length of stay in days per disease

\begin{tabular}{lccccccccc}
\hline Year & Asthma & Bronchiolitis & $\begin{array}{c}\text { Diabetes } \\
\text { Mellitus }\end{array}$ & Gastroenteritis & Pneumonia & $\begin{array}{c}\text { Meningitis } \\
\text { suspected }\end{array}$ & $\begin{array}{c}\text { Sepsis } \\
\text { suspected }\end{array}$ & $\begin{array}{c}\text { Sickle Cell } \\
\text { Disease }\end{array}$ & Seisures \\
\hline 2007 & 4.8 & 6.2 & 3.4 & 3.5 & 7.1 & 5.8 & 5.1 & 4.5 \\
2008 & 4 & 3.7 & 2.1 & 3.1 & 4.8 & 5 & 5.1 & 5 \\
2009 & 3.1 & 3.2 & 2.8 & 2.7 & 3.6 & 3.7 & 3.4 & 3.6 & 4.8 \\
2010 & 2.73 & 3.73 & 3.09 & 2.55 & 3.08 & 3.4 & 3.77 & 4.03 \\
2011 & 2.15 & 2.78 & 2.23 & 2.66 & 3.15 & 2.35 & 3.75 & 3.83 \\
\hline
\end{tabular}

